\title{
Risks and benefits of omega 3 fats for mortality, cardiovascular disease, and cancer: systematic review
}

Lee Hooper, Rachel L Thompson, Roger A Harrison, Carolyn D Summerbell, Andy R Ness, Helen J Moore, Helen V Worthington, Paul N Durrington, Julian P T Higgins, Nigel E Capps, Rudolph A Riemersma, Shah B J Ebrahim, George Davey Smith

\begin{abstract}
Objective To review systematically the evidence for an effect of long chain and shorter chain omega 3 fatty acids on total mortality, cardiovascular events, and cancer.

Data sources Electronic databases searched to February 2002; authors contacted and bibliographies of randomised controlled trials (RCTs) checked to locate studies.

Review methods Review of RCTs of omega 3 intake for $\geq 6$ months in adults (with or without risk factors for cardiovascular disease) with data on a relevant outcome. Cohort studies that estimated omega 3 intake and related this to clinical outcome during at least 6 months were also included. Application of inclusion criteria, data extraction, and quality assessments were performed independently in duplicate.

Results Of 15159 titles and abstracts assessed, 48 RCTs (36 913 participants) and 41 cohort studies were analysed. The trial results were inconsistent. The pooled estimate showed no strong evidence of reduced risk of total mortality (relative risk $0.87,95 \%$ confidence interval 0.73 to 1.03 ) or combined cardiovascular events $(0.95,0.82$ to 1.12$)$ in participants taking additional omega 3 fats. The few studies at low risk of bias were more consistent, but they showed no effect of omega 3 on total mortality $(0.98,0.70$ to 1.36$)$ or cardiovascular events $(1.09,0.87$ to 1.37). When data from the subgroup of studies of long chain omega 3 fats were analysed separately, total mortality $(0.86,0.70$ to $1.04 ; 138$ events) and cardiovascular events $(0.93,0.79$ to 1.11) were not clearly reduced. Neither RCTs nor cohort studies suggested increased risk of cancer with a higher intake of omega 3 (trials: 1.07, 0.88 to 1.30 ; cohort studies: $1.02,0.87$ to 1.19), but clinically important harm could not be excluded. Conclusion Long chain and shorter chain omega 3 fats do not have a clear effect on total mortality, combined cardiovascular events, or cancer.
\end{abstract}

\section{Introduction}

Consumption of long chain omega 3 fatty acids (eicosapentaenoic acid (EPA), docosapentaenoic acid (DPA), and docosahexaenoic acid (DHA)) found in fatty fish and fish oils has been linked to the low incidence of coronary heart disease in the Inuit people of Greenland ${ }^{1} ; \alpha$ linolenic acid (ALA), a shorter chain omega 3 found in some plant oils (and variably converted to eicosapentaenoic acid and docosahexaenoic acid) may also be protective. ${ }^{2}$ Omega 3 fats may protect against cardiovascular disease by lowering blood pressure and heart rate; reducing serum triglycerides, thrombotic tendency, inflammation, and arrhyth- mias; and improving endothelial function, insulin sensitivity, paraoxonase concentrations, and plaque stability. ${ }^{3-6}$

Toxic compounds, such as fat soluble methylmercury, dioxins, and polychlorinated biphenyls, are also found in oily fish and fish oils, but any harm from these compounds would be seen only after long term supplementation. ${ }^{78}$ Animal intervention studies and studies of adults after severe inadvertent exposure indicate that dioxins and polychlorinated biphenyls increase the risk of cancer. ${ }^{910}$ Methylmercury may increase the risk of myocardial infarction and cause neurological damage. ${ }^{11} 12$

Since a meta-analysis of the effect of omega 3 fats on cardiovascular morbidity and mortality in coronary heart disease suggested important benefits, ${ }^{13}$ a large intervention study has been published. $^{14}$ Our meta-analysis included these new data, balanced protective effects with possible harm, assessed the effects of plant based omega 3 fats on health, included people without established cardiovascular disease, and highlighted important questions about the role of omega 3 fats on cardiovascular disease and mortality. We systematically reviewed the effects of long chain and short chain omega 3 fats (together and separately) on mortality, cardiovascular disease, cancer, and bleeding events and analysed all relevant randomised controlled trials (RCTs) and prospective cohort studies.

\section{Methods}

The study methods have been described in detail elsewhere. ${ }^{15}$

Search strategy and study selection

We searched the Cochrane Library, Medline, Embase, the National Research Register, and SIGLE (to February 2002); we checked the bibliographies of included studies and contacted the authors. Articles not in English were translated. We excluded trials if they were not randomised; they had no omega $3 \mathrm{arm}$; the participants were children or were critically ill; the duration was $<6$ months; the intervention was multifactorial; or data on death, cardiovascular disease, or cancer were not available. We rejected cohort studies if they did not assess the intake of omega 3 , follow-up was $<6$ months, or the association between the intake of omega 3 and health was not investigated. Two reviewers assessed the inclusion of articles independently, and we contacted authors for more information on methodological quality, outcomes, and further studies.

A complete set of references is available on bmj.com 


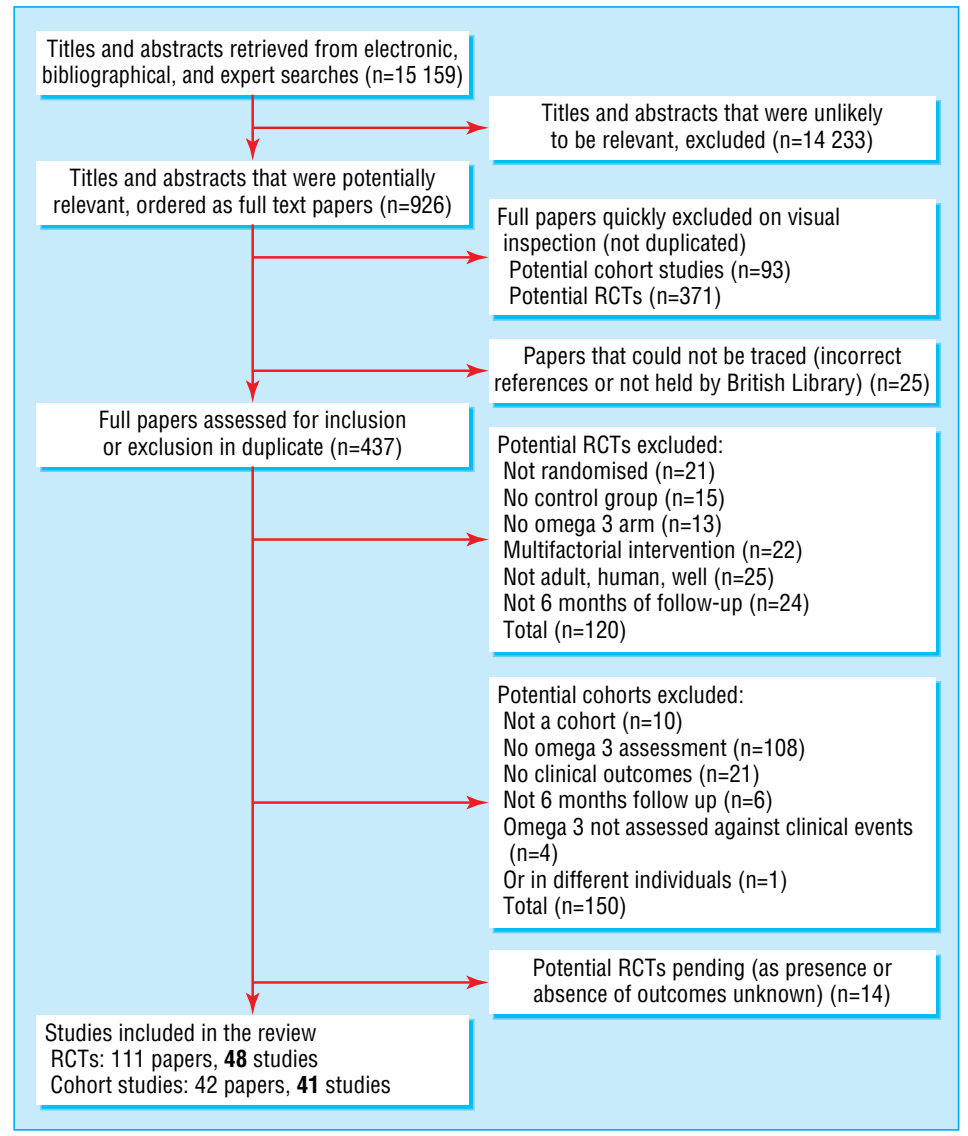

Fig 1 The selection process for randomised controlled trials (RCTs) and cohort studies on omega 3 fatty acid and health outcomes

\section{Data extraction and quality assessment}

Two reviewers independently extracted the data and assessed the quality of the studies. For RCTs we assessed the following quality characteristics: concealment of allocation to the study arms and the masking of participants, providers, and outcome assessors. We classed trials as having low risk of bias if allocation to the study arms was concealed and participants, providers, and outcome assessors were masked. Quality assessment of cohort studies was based on control group type, number lost to follow-up, baseline similarity, adjustment for dissimilarities, and masking.

\section{Data synthesis}

For RCTs we extracted the numbers of participants experiencing each outcome and total numbers randomised for each study arm, and combined by using relative risks in random effects meta-analysis. ${ }^{16}$ For cohort studies we used relative risk or odds ratio that had been adjusted for the most confounding factors, and we compared the most exposed quantile with the least exposed quantile. We used one analysis only for each cohort per outcome.

We used subgrouping of RCTs to explore the effects on mortality, cardiovascular events, and cancer of long chain versus short chain omega 3 fats and dietary advice versus supplementation. We used random effects meta-regression to analyse the effects of the dose of omega 3 and the duration of the trial. Sensitivity analyses assessed the robustness of RCT results to trial quality by restricting the analysis to studies with low risk of bias.

We used Cochran's test for heterogeneity to determine whether studies in a meta-analysis evaluated the same underlying sizes of effect. We used $\mathrm{I}^{2}$ to measure the degree of inconsistency among studies (the proportion of total observed variability due to genuine variation rather than random error within studies). ${ }^{17}$

\section{Results}

We screened 15159 titles and collected 926 full text papers. Forty eight randomised controlled trials and 41 analyses of 26

Table 1 Characteristics of trials and cohort studies on effects of omega 3 fats on mortality, cardiovascular disease (CVD), and cancer

\begin{tabular}{lcc}
$\begin{array}{l}\text { Criteria } \\
\text { Studies and participants }\end{array}$ & $\begin{array}{c}\text { Randomised } \\
\text { Controlled trials }\end{array}$ & Cohorts \\
\hline No of participants & 36913 & $563218, \begin{array}{c}\text { plus two where the size } \\
\text { not described }\end{array}$ \\
\hline No of studies & 48 & 47 published analyses from 26 \\
\hline No of large studies & $8(>500$ participants) & 10 (>20 000 participants $)$ \\
\hline Follow-up & 0.5 to $>\sim 6$ years & 4 to 25 years \\
\hline Risk of CVD & & 2 \\
\hline Have CVD & 21 & 2 \\
\hline Moderate risk & 10 & 22 \\
\hline Low risk & 17 & 14 \\
\hline Sex & & 4 \\
\hline$\geq 70 \%$ men & 24 & 7 \\
\hline $31 \%$ to $69 \%$ men & 17 & 1 \\
\hline$\leq 30 \%$ men & 5 & 13 \\
\hline Not stated & 2 & 10 \\
\hline Main outcome & & 10 \\
\hline CVD or mortality & 32 & \\
\hline Cancer & 0 & \\
\hline Other outcomes & 16 & \\
\hline
\end{tabular}

${ }^{*}$ Several cohorts appear to have more than one main outcome. 
Research

Table 2 Quality assessment of randomised controlled trials of the effect of omega 3 fats on mortality, cardiovascular disease, and cancer

\begin{tabular}{|c|c|c|c|c|c|c|}
\hline \multirow[b]{2}{*}{ Study } & \multirow[b]{2}{*}{$\begin{array}{l}\text { Concealment of } \\
\text { allocation }\end{array}$} & \multicolumn{3}{|c|}{ Masking } & \multirow[b]{2}{*}{$\begin{array}{l}\text { Dropouts for analysis of events (intervention, } \\
\text { control) }\end{array}$} & \multirow[b]{2}{*}{ Summary risk of bias } \\
\hline & & Participants & $\begin{array}{l}\text { Providers of } \\
\text { care }\end{array}$ & $\begin{array}{l}\text { Assessors of } \\
\text { outcome }\end{array}$ & & \\
\hline Almallah $1998^{w 1}$ & Done & Yes & Yes & Yes & $? / 18, ? / 18$ & Low \\
\hline$\overline{\text { Bairati } 1992^{\mathrm{w} 2}}$ & Done & Yes & Yes & Yes & $48 / 107,38 / 98$ & Low \\
\hline Bellamy 1992*w3 & Unclear & Unclear & Unclear & Unclear & $3 / 60,7 / 60$ & Medium or high \\
\hline Belluzzi $1996^{\text {w4 }}$ & Done & Yes & Yes & Yes & $5 / 39,2 / 39$ & Low \\
\hline Bemelmans $2002^{\mathrm{w} 5}$ & Done & Yes & Yes & Yes & $? / 51, ? / 52$ & Low \\
\hline Bonnema $1995^{\mathrm{w} 6}$ & Done & Yes & Yes & Yes & $0 / 14,1 / 14$ & Low \\
\hline Borchgrevink 1966 ${ }^{\text {w7 }}$ & Done & Yes & Yes & Yes & $? / 100, ? / 100$ & Low \\
\hline Brox 2001 ${ }^{\mathrm{w} 8}$ & Unclear & No & Yes & Yes & Seal oil $8 / 40$, cod liver oil $2 / 40,1 / 40$ & Medium or high \\
\hline Burr (DART) $1989^{w 9}$ & Unclear & No & No & Yes & 0/1015, 0/1018 for mortality & Medium or high \\
\hline Burr 2003 w10 & Unclear & No & No & Yes & 0/1571, 0/1543 for mortality & Medium or high \\
\hline Connor $1993^{\text {w11 }}$ & Done & Yes & Yes & Yes & $? / 8, ? / 8$ & Low \\
\hline Dehmer $1998^{\text {w12 }}$ & Not done & No & No & No & $3 / 46,5 / 44$ & Medium or high \\
\hline Dry $1991^{\text {w13 }}$ & Done & Yes & Yes & Yes & $0 / 6,0 / 6$ & Low \\
\hline Eritsland $1996^{\text {w14 }}$ & Done & No & No & Yes & $15 / 317,14 / 293$ & Medium or high \\
\hline Franzen $1993^{\text {w15 }}$ & Unclear & Yes & Unclear & Yes & $0 / 15,0 / 15$ & Medium or high \\
\hline Geusens $1994^{\star w 16}$ & Unclear & Yes & Yes & Yes & Low dose $9 / 30$, high dose $11 / 30,10 / 30$ & Medium or high \\
\hline GISSI-P 1999*w17 & Done & No & Unclear & Yes & $? / 5665, ? / 5658$ & Medium or high \\
\hline Greenfield $1993^{\text {w18 }}$ & Done & Yes & Yes & Yes & $3 / 16,1 / 8$ & Low \\
\hline Hawthorne $1992^{\text {w19 }}$ & Done & Unclear & Yes & Yes & $5 / 49,6 / 47$ & Medium or high \\
\hline Johansen 1999 *w20 & Unclear & Yes & Yes & Yes & $54 / 250,58 / 250$ & Medium or high \\
\hline Katan $1997^{\mathrm{w} 21}$ & Done & No & Unclear & Yes & $\begin{array}{c}\text { Low dose } ? / 15, \text { med dose ?/15, high dose } \\
? / 14, ? / 14\end{array}$ & Medium or high \\
\hline Kaul $1992^{\text {w22 }}$ & Unclear & No & No & Yes & $? / 58, ? / 49$ & Medium or high \\
\hline Lau $1993^{\text {w23 }}$ & Done & Yes & Yes & Yes & $9 / 32,16 / 32$ & Low \\
\hline Lau $1995^{\text {w24 }}$ & Done & Yes & Yes & Yes & $0 / 25,0 / 20$ & Low \\
\hline Leaf $1994^{\mathrm{w} 25}$ & Done & Yes & Yes & Yes & $69 / 275,69 / 276$ & Low \\
\hline Loeschke $1996^{\text {w26 }}$ & Done & Yes & Yes & Yes & $0 / 31,0 / 33$ & Low \\
\hline Lorenz-Meyer 1996²7 & Done & Yes & Yes & Yes & $? / 70, ? / 65$ & Low \\
\hline Malaguarnera 1999*w28 & Unclear & No & Unclear & Unclear & $? / 26, ? / 26$ & Medium or high \\
\hline Maresta $2002^{\text {w29 }}$ & Done & Yes & Yes & Yes & $44 / 169,38 / 170$ & Low \\
\hline Mate-Jimenez $1991^{\text {w30 }}$ & Done & No & Yes & Yes & $4 / 19,6 / 19$ & Medium or high \\
\hline Milner $1989^{\text {w31 }}$ & Done & No & Unclear & Yes & $0 / 100,0 / 100$ & Medium or high \\
\hline$\overline{\text { Natvig } 1968^{\star w 32}}$ & Done & Yes & Yes & Yes & $2 / 6716,2 / 6690$ & Low \\
\hline Nilsen $2001^{\text {w333 }}$ & Done & Yes & Yes & Yes & $? / 150, ? / 150$ & Low \\
\hline Nye $1990^{\star w 34}$ & Unclear & Yes & Yes & Yes & $0 / 36,0 / 37$ & Medium or high \\
\hline Reis $1991^{\text {w35 }}$ & Done & Yes & Yes & Yes & $22 / 146,10 / 72$ & Low \\
\hline Rossing $1996^{w 36}$ & Done & Yes & Yes & Yes & $0 / 18,0 / 18$ & Low \\
\hline Sacks (HARP) 1995*w37 & Unclear & Yes & Yes & Yes & $10 / 41,11 / 39$ & Medium or high \\
\hline Sacks (TOHP 1) 1994*w38 & Done & Yes & Yes & Yes & $1 / 175,1 / 175$ & Low \\
\hline Sarkkinen $1998^{\text {w39 }}$ & Unclear & No & No & Yes & $0 / 41,0 / 37$ & Medium or high \\
\hline Selvais $1995^{\text {w40 }}$ & Done & Yes & Yes & Yes & $4 / 12,2 / 12$ & Low \\
\hline Shimizu $1995^{\text {w41 }}$ & Not done & No & No & Yes & $? / 29, ? / 16$ & Medium or high \\
\hline Singh $1997 \dagger^{\text {w42 }}$ & Done & No & Yes & Yes & Fish oil 4/122, mustard oil 8/120, 6/118 & Medium or high \\
\hline Sirtori $1998^{\text {w43 }}$ & Done & Yes & Yes & Yes & $28 / 470,39 / 465$ & Low \\
\hline Skoldstam $1992^{\text {w44 }}$ & Done & No & No & No & $1 / 23,2 / 23$ & Medium or high \\
\hline Terano $1999^{\text {w45 }}$ & Done & No & No & Yes & $0 / 10,0 / 10$ & Medium or high \\
\hline Thien $1993^{\mathrm{w} 46}$ & Done & Yes & Yes & Yes & $6 / 21,6 / 16$ & Low \\
\hline Veale $1994^{\text {w47 }}$ & Done & Yes & Yes & Yes & $4 / 19,0 / 19$ & Low \\
\hline von Schacky $1999^{\text {w48 }}$ & Done & Yes & Yes & Yes & $? / 112, ? / 111$ & Low \\
\hline
\end{tabular}

Allocation concealment was coded as done, unclear, or not done; blinding of participants, providers, and outcome assessors was coded as yes (where there was a clear and realistic attempt to blind-success of blinding was rarely checked in the studies), unclear, or no (in consultation with authors of trials where possible).

A trial was considered to be at low risk of bias if allocation concealment was "done" and blinding of participants, providers, and outcome assessors was coded "yes." All other trials were considered at moderate or high risk of bias.

*Did not respond to our request for further information on the quality of their studies or provide additional data on trial outcomes.

†The BMJ has concerns about the validity of another paper written by this author. ${ }^{18}$

cohort studies fulfilled all inclusion criteria (fig 1 ; for a complete set of references see bmj.com). Table 1 shows the main characteristics of the included studies.

\section{Intervention or exposure}

Dietary supplements were given in 44 trials (36 as capsules, six as oil, one each as liquid emulsion and enriched margarine), advice on eating oily fish in three, and advice on diet and food supplements in one. Supplements were long chain omega 3 fats (usually whole or concentrated fish oil; one small trial used refined eicosapentaenoic acid and one used refined docosahexaenoic acid), and five studies provided shorter chain omega 3 fats. Doses of long chain omega 3 fats (summing eicosapentaenoic acid, docosahexaenoic acid, and docosapentaenoic acid) varied from $0.4 \mathrm{~g}$ to $7.0 \mathrm{~g}$ per day. Control groups received vegetable oils, other fats, "inert" or ill defined substances, different dietary advice, or nothing. The intervention lasted 6-11 months 
Table 3 Adjustment carried out in 10 cohorts that assessed effects of omega 3 fats on cancer

\begin{tabular}{ll} 
Factors* & No of cohorts adjusted† \\
\hline Lifestyle factors & \\
\hline Smoking & 2 \\
\hline Alcohol intake & 2 \\
\hline Physical activity & 1 \\
\hline Dietary fibre intake & 1 \\
\hline Saturated fat intake & 1 \\
\hline Trans fat intake & 0 \\
\hline Red meat intake & 2 \\
\hline Fruit intake & 0 \\
\hline Vegetable intake & 0 \\
\hline Otherst: &
\end{tabular}

\begin{tabular}{lc} 
Othersł: & \\
\hline Body mass index & 4 \\
\hline Total energy intake & 5 \\
\hline $\begin{array}{l}\text { Other dietary factors, height, parity, and menopausal } \\
\text { status }\end{array}$ & 1 each \\
\hline
\end{tabular}$$
\text { status }
$$

Social factors

\begin{tabular}{ll}
\hline Place of residence & 2 \\
\hline Education & 2 \\
\hline
\end{tabular}

\begin{tabular}{ll} 
Education & 2 \\
\hline Otherf: & \\
\hline
\end{tabular}

\begin{tabular}{ll}
\hline Ethnic origin & 1 \\
\hline Socioeconomic status & 1 \\
\hline
\end{tabular}

\section{Interest in health}

\begin{tabular}{ll}
\hline Vitamin E supplementation & 0 \\
\hline Multivitamin supplementation & 0
\end{tabular}

Othersł:

\begin{tabular}{ll}
\hline Use of hormone replacement therapy & 1 \\
\hline Family history of the disease studied & 2 \\
\hline Precancerous symptoms & 1
\end{tabular}

${ }^{*}$ Factors that differ consistently between people taking most and least fish based omega 3 fats; the association was consistent across all cohorts that assessed it and was seen in at least two cohorts

†The number of cohorts that adjusted for the factor in the most adjusted analysis, of the 10 cohorts used in the cancer outcomes analysis. Six cohorts also adjusted for age. fUnclear if consistently associated with omega 3 fats.

in 23 studies, 12-23 months in 16, 24-47 months in eight, and $\geq 48$ months in one study.

Intake of omega 3 (varying combinations of eicosapentaenoic acid, docosahexaenoic acid, docosapentaenoic acid, along with $\alpha$ linolenic acid, supplemental fish oils, or dietary oily fish) was assessed by dietary and biochemical means in two cohorts, dietary means only in 18, and biochemical means only in 10 . Groups with the lowest and highest intake of long chain omega 3 differed by 0.1-0.6 $\mathrm{g}$ omega 3 per day.

\section{Methodological quality}

Twenty five RCTs were rated as having a low risk of bias (table 2). Losses to follow-up were unclear in 16 cohort studies. In 15 cohort studies the outcome assessors were blinded to exposure, in two they were not blinded, and in nine blinding was unclear.

In the seven cohort studies that described omega 3 intake at baseline (five assessed long chain omega 3 fats only, one short chain omega 3 fats only, and one assessed both), the characteristics of participants with high and low intake of omega 3 fats differed. People who consumed most long chain omega 3 at baseline had an advantage with regard to lifestyle (smoking, diet, and exercise), interest in health, and social factors (education, living in town). Adjustment for these potential confounding factors may not have been adequate (table 3 ).

\section{Total mortality}

Deaths occurred in 15 RCTs (1995 deaths), and authors of 29 RCTs reported that no deaths occurred. Evidence that risk of death was reduced in participants randomised to omega 3 (rela- tive risk $0.87,95 \%$ confidence interval 0.73 to 1.03 ) was weak, and inconsistency was moderate $\left(\mathrm{I}^{2}=42 \%\right)$ (fig 2$)$. When analysis was restricted to studies at low risk of bias this effect was attenuated $(0.98,0.70$ to $1.36 ; 138$ deaths), and inconsistency between RCTs was low $\left(\mathrm{I}^{2}=0 \%\right)$. This sensitivity analysis removed the RCT by Singh, whose studies have been questioned. ${ }^{18}$

Results were similar for long chain versus short chain omega 3 (fig 2) and dietary advice versus supplements (data not shown). Meta-regression indicated that the risk of death increased as the length of the RCT increased (regression coefficient 0.008, 0.003 to 0.012 ). This is compatible with omega 3 fats having an early protective effect that later becomes harmful; however, the association was lost when we removed the large trial by Burr et al. ${ }^{14}$ Meta-regression did not suggest a relation between mortality and the dose of long chain omega 3. Cohort studies suggested that omega 3 protected against death $(0.65,0.48$ to 0.88 ; $\mathrm{I}^{2}=36 \%$ ), but it was unclear whether adjustment for confounders was adequate.

\section{Combined cardiovascular events}

Eighteen RCTs provided data on cardiovascular events in 2628 participants. The meta-analysis showed no definite effect of omega 3 fats on cardiovascular events, but confidence intervals were wide $(0.95,0.82$ to 1.12$)$ and inconsistency was high $\left(\mathrm{I}^{2}=65 \%\right)$ (fig 3). Removing studies at moderate or high risk of bias reduced but did not remove inconsistency $(1.09,0.87$ to $1.37 ; 570$ events; $\mathrm{I}^{2}=49 \%$ ).

Subgrouping by long chain versus short chain omega 3 or by advice to eat oily fish versus supplements did not generate robust effects of omega 3 fats on cardiovascular events. Cohort studies provided no strong evidence that omega 3 fats protect against cardiovascular events.

\section{Cancer}

Ten RCTs reported the incidence of cancer; 391 diagnoses of cancer or death from cancer occurred in 17433 participants (two of the trials reported no cancers). We found no evidence that omega 3 fats had an effect on the incidence of cancer (1.07, 0.88 to 1.30$)$ and there was no inconsistency $\left(I^{2}=0 \%\right)($ fig 4$)$. Five trials and seven events remained on sensitivity analysis.

Seven cohort studies provided data on cancer (832 events in the highest and lowest quantiles), and meta-analysis found no effect of high versus low intake of omega 3 (1.02, 0.87 to 1.19 ; $\left.\mathrm{I}^{2}=21 \%\right)$.

\section{Outcomes related to bleeding}

Nine RCTs reported at least one stroke (243 strokes in total), but little information was available specifically on haemorrhagic stroke. Omega 3 had no clear effect on the total numbers of strokes $\left(1.17,0.91\right.$ to $\left.1.51 ; \mathrm{I}^{2}=0 \%\right)$, in sensitivity analysis (29 events), or in four cohort studies (0.87, 0.72 to 1.04 ) (fig 5).

\section{Discussion}

Our meta-analysis of RCTs assessing the effects of increased omega 3 fats on total mortality found substantial variations between studies. Studies with stronger methodology had more consistent results, and the pooled relative risk of these studies was 0.98 ( 0.70 to $1.36 ; 138$ events). We found no evidence from RCTs or cohort studies that omega 3 fats have an effect on combined cardiovascular events. Neither RCTs nor cohort studies showed significantly increased risks of cancer or stroke with higher intake of omega 3 , but there were too few events to rule out important effects. 


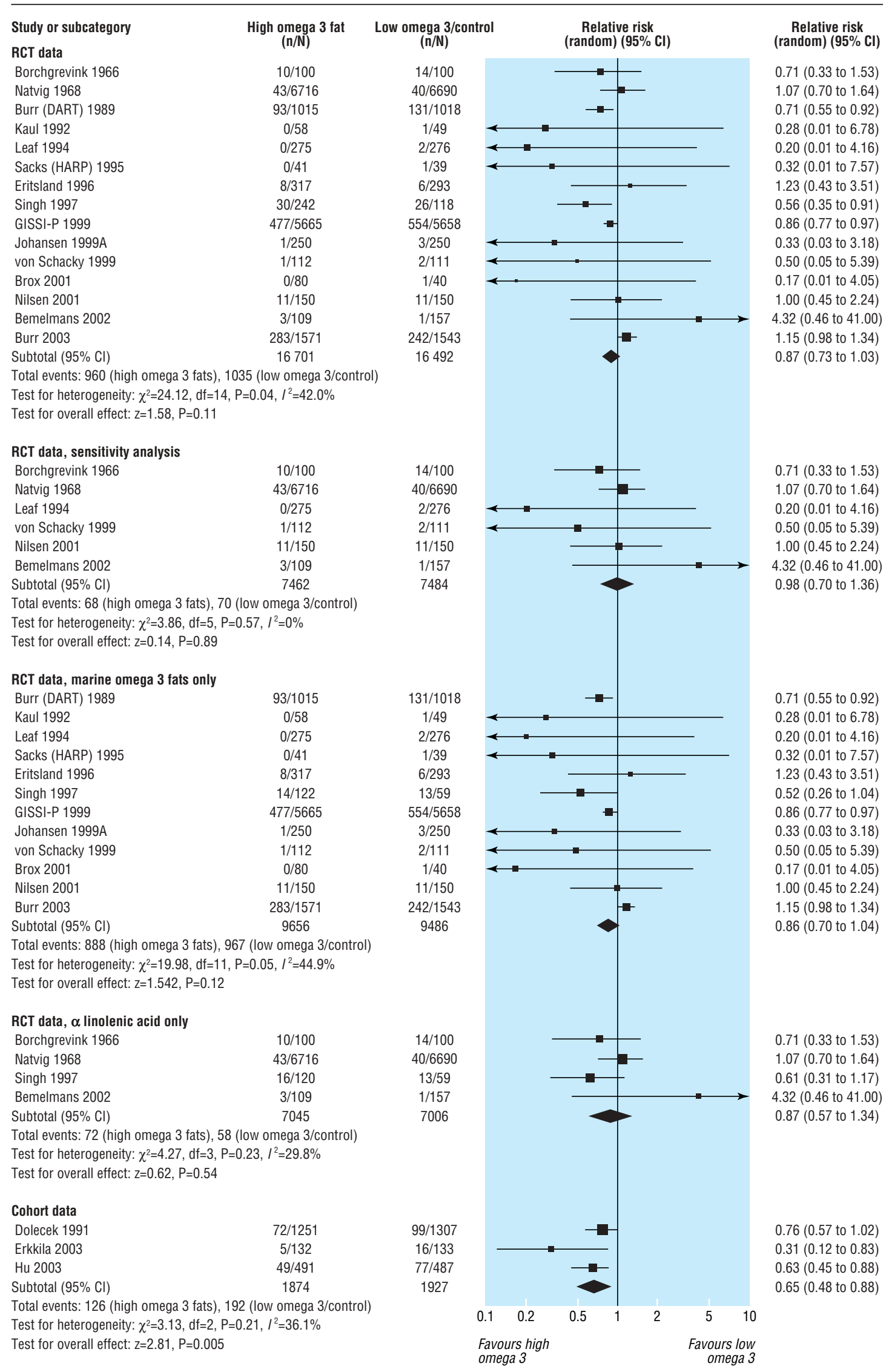

Fig 2 Effect of omega 3 fatty acids on mortality. For references see bmj.com 


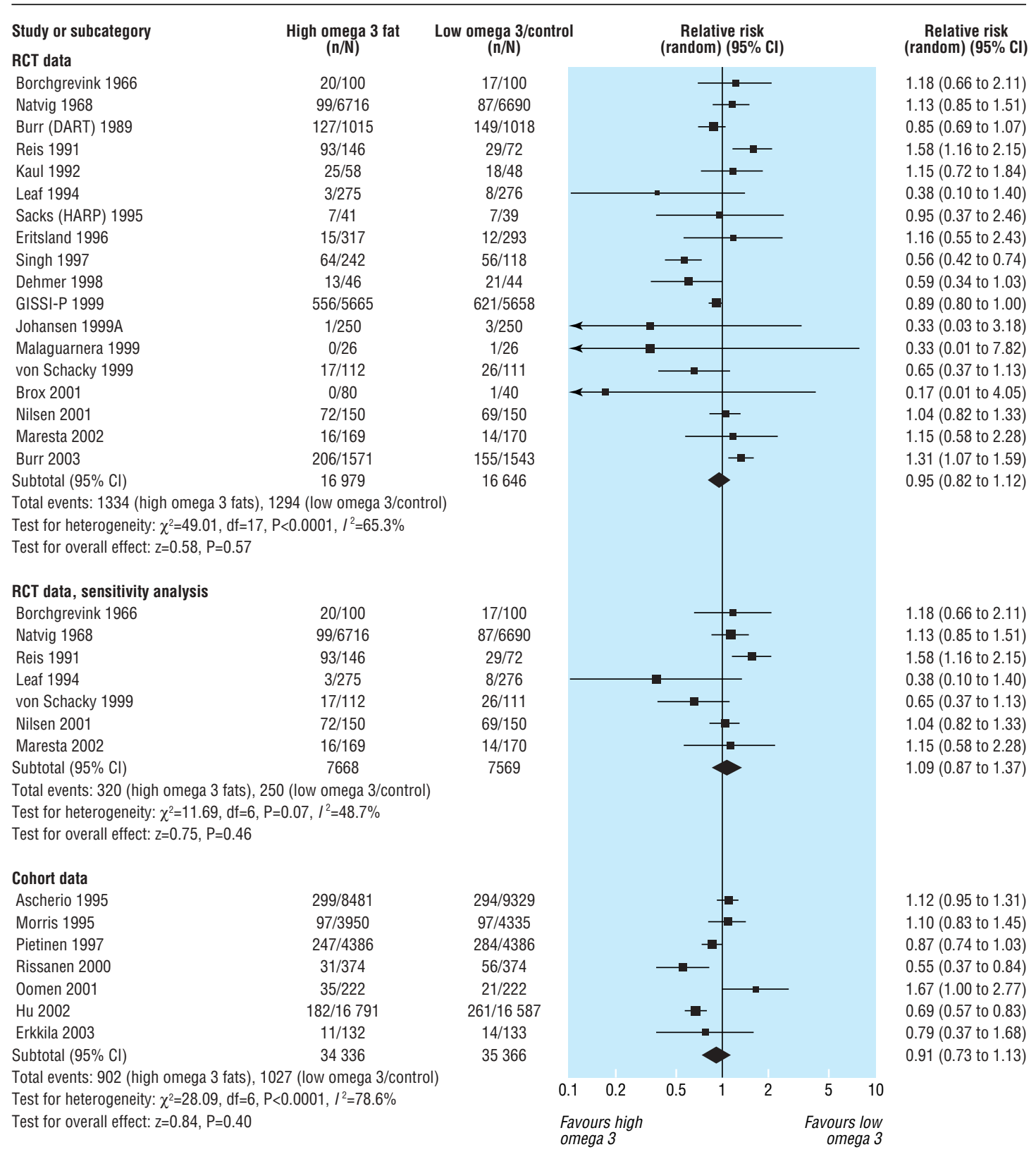

Note: Borchgrevink 1966, Natvig 1968, and 120 participants from Singh 1997 received short chain (not long chain) omega 3 fats.

Trials of long chain omega 3 fats only had a pooled relative risk of cardiovascular events of $0.93(95 \% \mathrm{Cl} 0.79$ to 1.11$)$, and trials of short chain omega 3 only had a pooled relative risk of 0.92 (0.58 to 1.45)

Fig 3 Effect of omega 3 fatty acids on cardiovascular events. For references see bmj.com

\section{Strengths and weaknesses}

The largest studies reviewed had greater potential for bias than some of the smaller ones. We hoped that pooling studies at low risk of bias might provide enough power to inform us of effects on health, but this was not the case (only 138 deaths and 570 cardiovascular events). Similarly, analysis of the effects of omega 3 on rarer outcomes such as stroke had insufficient power to detect clinically important effects. Unlike previous metaanalyses, we reviewed systematically the effects of omega 3 fats on mortality, cardiovascular disease, cancer, and bleeding events and analysed all relevant RCTs and prospective cohort studies. We also accounted for differences in study quality and examined the effects of long chain and short chain omega 3 fats in a wide group of participants; this provides high quality evidence to guide policy and practice.

\section{Other studies}

Our findings differ from those of a recent systematic review by Bucher et al, ${ }^{13}$ which reviewed trials assessing the effects of long chain omega 3 fats over at least six months in patients with coronary heart disease and found significant protection from mortality $(0.8,0.7$ to 0.9$)$ and sudden death $(0.7,0.6$ to 0.9$)$. Bucher et al analysed 11 RCTs (nine included here, two excluded as multifactorial interventions) with 15806 participants and 1335 deaths but did not include the recent study by Burr et al with 3114 participants included in our study. ${ }^{14}$ 


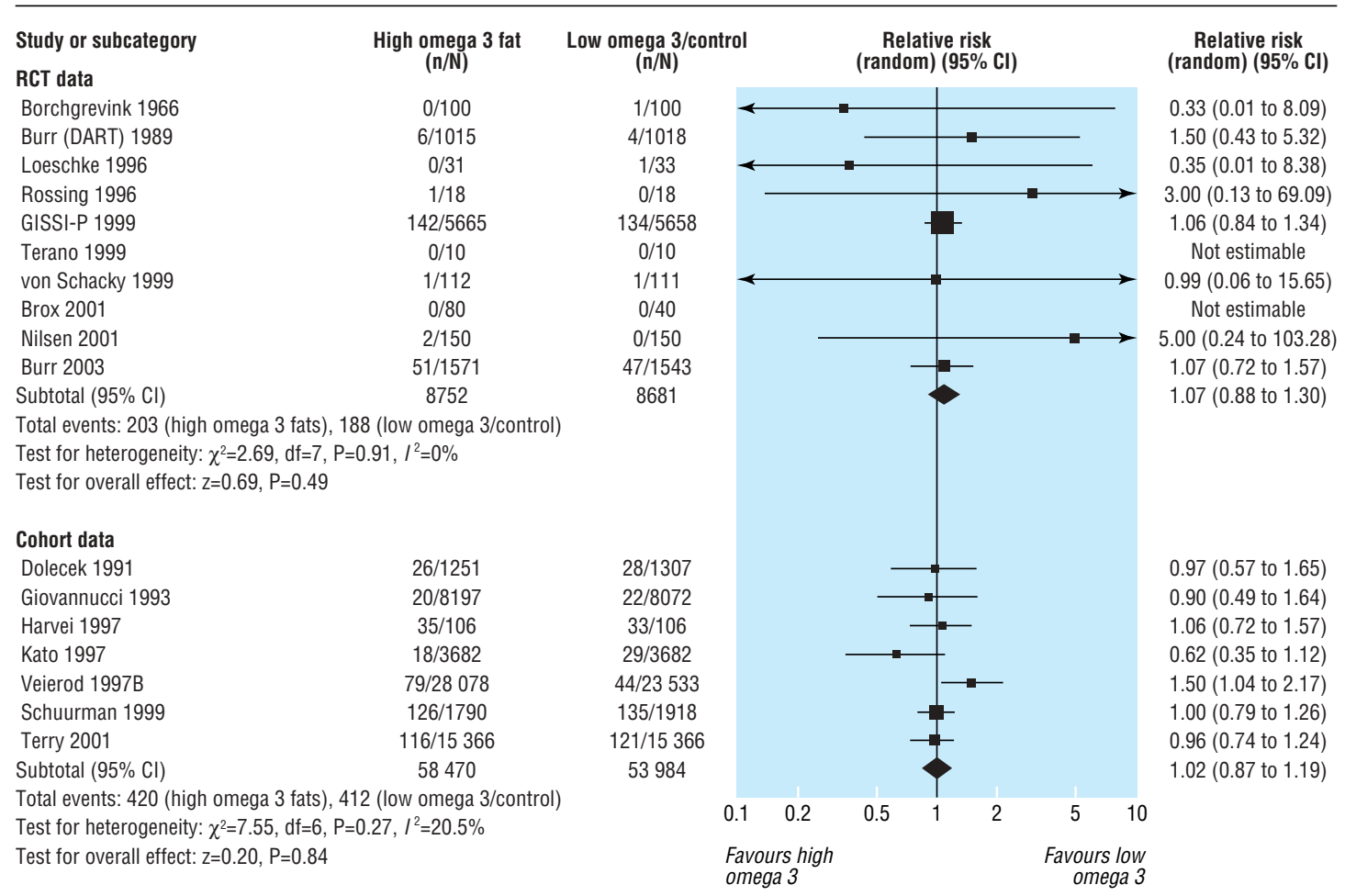

Note: Participants in Borchgrevink 1966 received short chain omega 3 fats (the remaining trials provided long chain omega 3 fats).

Trials of long chain omega 3 fats only had a pooled relative risk of cancer of 1.08 (95\% $\mathrm{Cl} 0.88$ to 1.31$)$

Fig 4 Effect of omega 3 fatty acids on the diagnosis of cancer or death from cancer. For references see bmj.com

A systematic review from the United States collated RCTs and cohort studies to assess the effects of marine omega 3 fats, but this study did not perform meta-analysis, reported the results of the largest studies only, and did not investigate reasons for conflicting results. ${ }^{19}$ A recent review from the United Kingdom of the benefits and risks of consumption of fish on cardiovascular

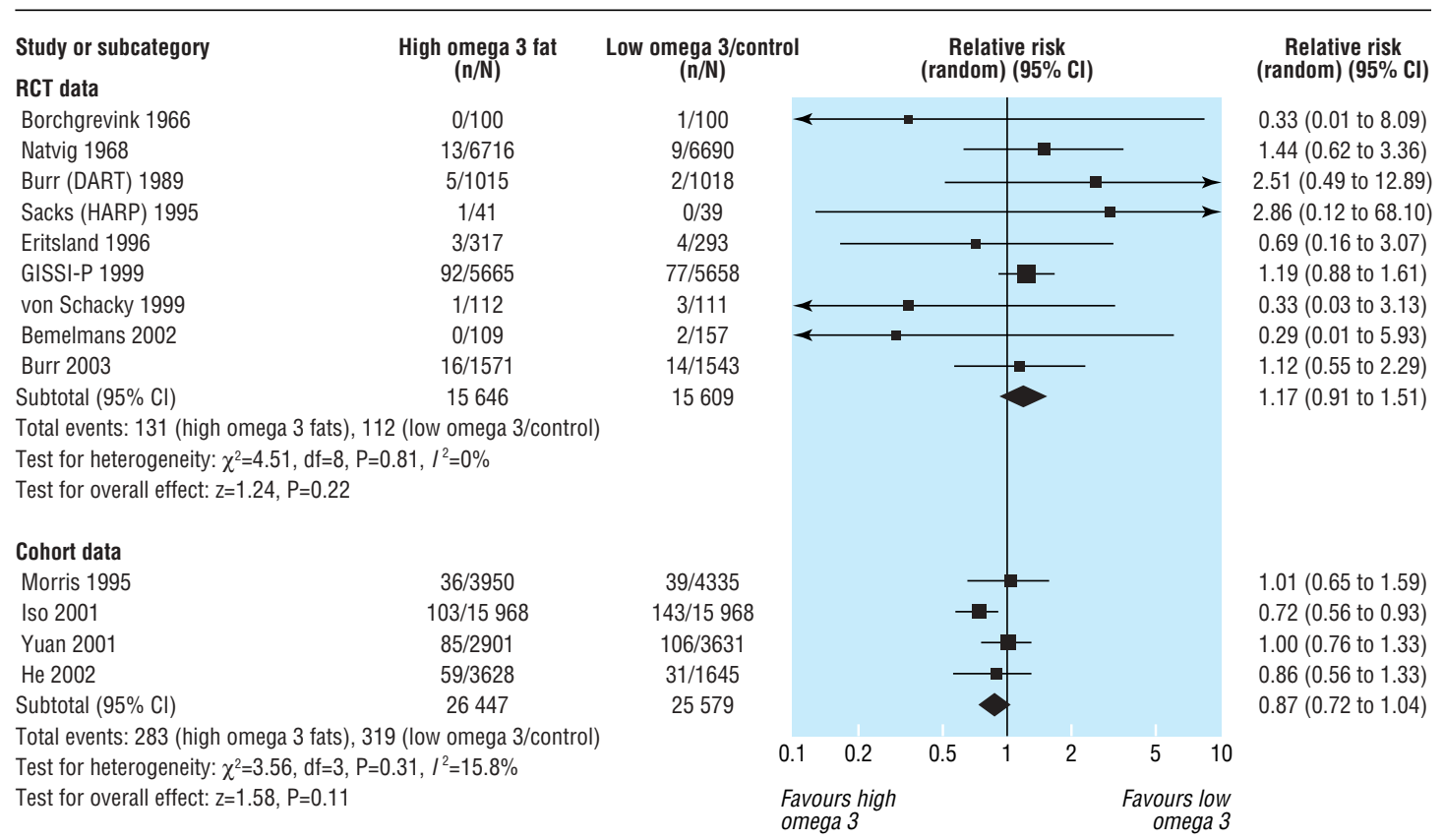

Note: Participants in Borchgrevink 1966, Natvig 1968, and Bemelmans 2002 received short chain omega 3 fats, participants in the other trials received long chain omega 3 fats. Trials of long chain omega 3 fats only had a pooled relative risk of stroke of $1.17(95 \% \mathrm{Cl} 0.90$ to 1.53$)$, and trials of short chain omega 3 fats only had a pooled relative risk of $1.18(0.53$ to 2.60$)$

Fig 5 Effect of omega 3 fatty acids on stroke. For references see bmj.com 
disease mentioned only four large trials and did not pool the results. $^{20}$

Our inclusion of studies with short chain omega 3 as the active intervention or with participants who had a lower risk of cardiovascular disease does not explain the differences between our results and those of Bucher et al. When we pooled studies in which participants were given only long chain omega 3 fats or were at high risk of cardiovascular disease the risk of death was not significantly reduced. However, when we removed the study by Burr et al from our meta-analysis, risk of death was similar to that reported by Bucher et al $(0.83,0.75$ to 0.91$)$. The study by Burr et al (included in our review as an unpublished study, since published) is the second largest study in terms of deaths reported (525 deaths; the GISSI-P study reports 1031 deaths). ${ }^{21}$

Why does the study by Burr et al contradict the other large studies by not suggesting a benefit of omega $3 ?^{14}$ The first possible explanation is that this RCT had the longest follow-up of all RCTs and the harmful effects of methylmercury could be cumulative. Time course analysis of the GISSI-P trial was consistent with the risk of death rising over the 42 months of the trial, but the increase in mortality was seen within the first year in the study by Burr et al. ${ }^{1422}$ A second explanation is that the study by Burr et al was the only RCT that specifically enrolled men treated for angina. Post hoc subgroup analysis of the GISSI-P trial indicates that people with heart failure benefited most from omega 3 supplements (R Marchioli, personal communication, 2004). As heart failure is more common after myocardial infarction this may explain an attenuation of effect but would not explain the increase in risk suggested in the study by Burr et al. A third possibility is that omega 3 from oily fish has a different effect to fish oil supplements, but this was investigated by Burr et al and found not to explain the differences. It is therefore not clear why the results of Burr et al differ from the other large studies on fish based omega 3. It is possible that performance bias due to differential care in the intervention and control arms occurred in trials where the intervention was not masked, including GISSI-P and the Burr study, ${ }^{14}{ }^{21}$ but dietary intake, other relevant risk factors, and pharmacotherapy appeared similar in both arms of these trials. It may be that the effect of omega 3 fats on cardiovascular disease is smaller than previously thought, or that its beneficial effect is limited to a specific group (such as patients after myocardial infarction or with heart failure) not represented in the study by Burr et al. ${ }^{14}$

\section{Cohort studies or RCTs?}

A recent meta-analysis assessed the effects of consumption of fish on stroke in cohort studies and found that people who ate white or oily fish at least once a week had a significantly reduced risk of stroke. ${ }^{24}$ We excluded cohort studies that assessed only total fish intake (as this is not clearly related to omega 3 intake). The web of lifestyle, interest in health, and social factors (health patterning) seen in the cohort studies included in our review provides an advantage to people taking most long chain omega 3 fats, and this makes adequate adjustment for confounding difficult, if not impossible. Thus, we must rely on high quality RCTs to provide non-confounded answers about the effects of omega 3 fats on health. Some effects of fish on health may be due to components other than omega 3-for example, selenium or vita$\min \mathrm{D}$.

\section{Interpretation}

It is not clear whether long chain or short chain omega 3 fats (together or separately) reduce or increase total mortality, cardiovascular events, cancer, or strokes. Our findings do not rule out an important effect of omega 3 fats on total mortality, as
What is already known on this topic

A systematic review of randomised controlled trials in coronary heart disease showed reduced mortality in patients taking supplemental long chain omega 3 fats

\section{What this study adds}

This systematic review assessed the health effects of long chain and shorter chain omega 3 fats (together or separately) on total mortality, cardiovascular events, cancer, and strokes in a wide group of participants and found no evidence of a clear benefit of omega 3 fats on health

robust trials at low risk of bias reported few deaths. There is no evidence that the source (dietary or supplemental) and dose of omega 3 fats affected the effectiveness of long chain omega 3 fats.

UK guidelines encourage the general public to eat more oily fish, and higher amounts are advised after myocardial infarction (supported by trials after myocardial infarction). ${ }^{20}{ }^{25}{ }^{26}$ This advice should continue at present but the evidence should be reviewed regularly. It is probably not appropriate to recommend a high intake of omega 3 fats for people who have angina but have not had a myocardial infarction.

Adjustment for lifestyle factors appeared to be inadequate in the cohort studies, so policy and lifestyle decisions should be based on data from RCTs. To understand the effects of omega 3 fats on health, we need more high quality RCTs (with adequate concealment of allocation and masking of participants and health providers) of long duration that also report the associated harms.

Thanks to Theresa Moore and Margaret Burke from the Cochrane Heart Group, and to all of the authors of primary studies who helped us build up the data. This paper is based on a Cochrane review accepted for publication in The Cochrane Library (see www.TheCochraneLibrary.net for information).

Contributors: All authors commented critically on the manuscript and agreed the final version. NEC helped design the review, provided a clinical perspective, and commented on the analysis and interpretation. GDS helped design the review, provided a methodological perspective and general advice, and commented on the analysis and interpretation. PND provided a methodological perspective and expertise on omega 3 biochemistry, and commented on the analysis and interpretation. SBJE helped design the review, provided a methodological perspective and general advice, and commented on the analysis and interpretation. RAH screened retrieved papers against inclusion criteria, appraised quality of papers, abstracted data from papers, provided general advice, and commented on the analysis and interpretation. JPTH helped design the review, provided a statistical perspective and general advice, and commented on the analysis and interpretation. $\mathrm{LH}$ conceived the review, designed and coordinated the review, developed the search strategy and undertook searches, screened the search results, organised retrieval of papers, screened retrieved papers against inclusion criteria, appraised study quality, abstracted data from included papers, wrote to authors and experts for additional information, managed the review data, entered data into RevMan, analysed and interpreted the data, and was the primary author. HJM screened retrieved papers against inclusion criteria, appraised quality of papers, abstracted data from papers and commented on the analysis and interpretation, ARN commented on the protocol, provided additional relevant articles, screened retrieved papers against inclusion criteria, appraised quality of papers, abstracted data from papers, and was involved in discussing the findings, interpreting the data, and writing up. RAR helped design the review, screen retrieved papers against inclusion criteria, appraise quality of papers, and interpret the data, provided general advice, and commented on the analysis and interpretation. CDS helped design the review, screen retrieved papers against inclusion criteria, appraise quality of papers, and abstract data from papers, and commented on the analysis and interpretation. RLT helped design the review, screen retrieved papers against inclusion criteria, appraise quality of papers, abstract data from 
papers, provide general advice, and comment on the analysis and interpretation. HVW helped in the analysis and interpretation of the data and provided a methodological and statistical perspective and general advice. LH is guarantor.

Funding: A northwest research and development research fellowship (UK Department of Health) and the British Dietetic Association.

Competing interests: NC has received fees for speaking by Solvay Healthcare, who market Omacor

Ethical approval: Not required.

1 Bang HO, Dyerberg J. Plasma lipids and lipoproteins in Greenlandic west coas Eskimos. Acta Med Scand 1972;192:85-94

2 Nettleton JA. Omega-3 fatty acids: comparison of plant and seafood sources in human nutrition.J Am Diet Assoc 1991;91:331-7.

3 Bhatnagar D, Durrington PN. Omega-3 fatty acids: their role in the prevention and treatment of atherosclerosis related risk factors and complications. Int J Clin Pract 2003;57:305-14.

4 Thies F, Garry JM, Yaqoob P, Rerkasm K, Williams J, Shearman CP, et al. Association of n-3 polyunsaturated fatty acids with stability of atherosclerotic plaques: a randomised controlled trial. Lancet 2003:361:477-85.

5 Geelen A, Brouwer IA, Zock PL, Katan MB. Antiarrhythmic effects of n-3 fatty acids: evidence from human studies. Curr Opin Lipidol 2004;15:25-30.

6 Din JN, Newby DE, Flapan AD. Omega 3 fatty acids and cardiovascular disease-fishing for a natural treatment. BMJ 2004;328:30-5

7 Food Standards Agency. Dioxins and PCBs in the UK diet: 1997. Total diet study samples. London: Food Standards Agency, 2000. www.foodrisk.org/dioxin_pcb_exposure.cfm (accessed 13 Mar 2006)

8 Ministry of Agriculture, Fisheries and Food. Concentrations of metals and other elements in marine fish and shellfish. London: MAFF, 1998. archive.food.gov.uk/maff/archive/food/ infsheet/1998/no166/166vege.htm (accessed 13 Mar 2006).

9 Liem AKD, Theelen RMC. Dioxins:chemical analysis, exposure and risk assessment. Utrecht: Liem AKD, Theelen RMC. D
Universiteit Utrecht, 1997.

10 Steenland K, Bertazzi P, Baccarelli A, Kogevinas M. Dioxin revisited: developments since the 1997 IARC classification of dioxin as a human carcinogen. Environ Health Perspect 2004;112:1265-8.

11 Guallar E, Sanz-Gallardo I, van't Veer P, Bode P, Aro A, Gomez-Aracena J, et al. Mercury, fish oils, and the risk of myocardial infarction. N Engl J Med 2002;347:174653.

12 Risher JF, Murray HE, Prince GR. Organic mercury compounds: human exposure and its relevance to public health. Toxicol Ind Health 2002;18:109-60.

13 Bucher HC, Hengstler P, Schindler C, Meier G. N-3 polyunsaturated fatty acids in coronary heart disease: a meta-analysis of randomized controlled trials. Am J Med

14 Burr ML, Ashfield-Watt PA, Dunstan FD, Fehily AM, Breay P, Ashton T, et al. Lack of benefit of dietary advice to men with angina: results of a controlled trial. Eur J Clin Nutr 2003:57:193-200

15 Hooper L, Thompson RL, Harrison RA, Summerbell CD, Moore H, Worthington HV et al. Omega 3 fatty acids for prevention and treatment of cardiovascular disease. Cochrane Database Syst Rev 2004;4:CD003177.

16 DerSimonian R, Laird N. Meta-analysis in clinical trials. Control Clin Trials 1986;7:17788.

17 Higgins JPT, Thompson SG, Deeks JJ, Altman DG. Measuring inconsistency in meta-analysis. BMJ 2003;327:557-60.

18 Expression of concern. BMJ 2005:331:266.

Wang C, Chung M, Balk E, Kupelnick B, DeVine D, Lawrence A, et al. Effects of omegafatty acids on cardiovascular disease. Rockville, MD, USA: Agency for Healthcare Research and Quality, 2004. Evidence Report/Technology Assessment 94 www.ncbi.nlm.nih.gov/books/bv.fcgi?rid = hstat1a.chapter.38290 $\quad$ (accessed 13 Mar 2006)
20 Scientific Advisory Committee on Nutrition. Advice on fish consumption: benefits and risks London: Stationery Office, 2004. www.food.gov.uk/multimedia/pdfs/ fishreport200401.pdf (accessed 13 Mar 2006)

21 GISSI-Prevenzione Investigators. Dietary supplementation with n-3 polyunsaturated fatty acids and vitamin $\mathrm{E}$ after myocardial infarction: results of the GISSI-Prevenzione trial. Lancet 1999;354:447-55.

22 Marchioli R, Barzi F, Bomba E, Chieffo C, Di Gregorio DDMR, Franzosi MG, et al. Early protection against sudden death by n-3 polyunsaturated fatty acids after myocardial infarction: time course analysis of the results of the Gruppo Italiano per lo Studio della Sopravvivenza nell'Infarto Miocardico (GISSI)-Prevenzione Circulation 2002;105:1897-903.

23 Burr ML, Fehily AM, Gilbert JF, Rogers S, Holliday RM, Sweetnam PM, et al. Effects of changes in fat, fish, and fibre intakes on death and myocardial reinfarction: diet and changes in fat, fish, and fibre intakes on death
reinfarction trial (DART). Lancet 1989;2:757-61.

24 He K, Song Y, Daviglus ML, Liu K, Van Horn L, Dyer AR, et al. Fish consumption and incidence of stroke: a meta-analysis of cohort studies. Stroke 2004;35:1538-42.

25 Wood D, Durrington P, Poulter N, McInnes G, Rees A, Wray R, et al. Joint British recommendations on prevention of coronary heart disease in clinical practice. Heart 1998;80:S1-29.

26 Scottish Intercollegiate Guidelines Network. Cardiac rehabilitation: a national clinical guideline. Edinburgh: SIGN, 2002. www.sign.ac.uk/guidelines/fulltext/57/index.html (accessed 24 Feb 2006).

(Accepted 1 February 2006)

doi $10.1136 /$ bmj.38755.366331.2F

School of Medicine, Health Policy and Practice, University of East Anglia, Norwich NR4 7TJ

Lee Hooper lecturer

Institute of Human Nutrition, University of Southampton, Southampton SO16 $6 \mathrm{YD}$

Rachel L Thompson senior research fellow

Bolton Primary Care Trust, Bolton BL1 1PP

Roger A Harrison senior research fellow

School of Health and Social Care, University of Teesside, Middlesbrough TS1 3BA Carolyn D Summerbell professor

Helen J Moore research fellow

Department of Community-based Medicine, University of Bristol, Bristol BS8 1TQ Andy R Ness senior lecturer

Department of Social Medicine, University of Bristol, Bristol BS8 2PR

George Davey Smith professor

School of Dentistry, University of Manchester, Manchester M15 6FH

Helen V Worthington professor

Department of Medicine, University of Manchester

Paul N Durrington professor

MRC Biostatistics Unit, Cambridge CB2 2SR

Julian P T Higgins statistician

Department of Clinical Biochemistry, Princess Royal Hospital, Shrewsbury and Telford Hospital NHS Trust, Telford TF1 6TF

Nigel E Capps consultant chemical pathologist

Centre for Cardiovascular Science, University of Edinburgh, Edinburgh EH16 4TJ Rudolph A Riemersma professor

London School of Hygiene and Tropical Medicine, London WC1E 7HT

Department of Epidemiology and Population Health

Shah B J Ebrahim professor

Correspondence to: L Hooper 1.hooper@uea.ac.uk 